\title{
Fluorescent Dye LS301
}

National Cancer Institute

\section{Source}

National Cancer Institute. Fluorescent Dye LS301. NCI Thesaurus. Code C129590.

\begin{abstract}
A hydrophobic near-infrared (NIR) fluorescent probe composed of a NIR fluorescent dye cypate linked to an unnatural D-cysteine-containing cyclic octapeptide (D-Cys-Gly-Arg-Asp-Ser-Pro-Cys)-Lys-OH, with potential cancer imaging activity. Upon administration during tumor resection, the peptide component of LS301 specifically targets and binds integrin receptors expressed on tumor cells, which are not expressed on normal, healthy cells. Upon receptor-mediated endocytosis and fluorescence imaging using infrared light that is visualized by the surgeon with special Cancer Vision Goggles (CVG), tumor cells can be detected, identified, and removed accordingly. This enhances the accuracy of cancer cell removal during surgery. The D-cysteine on the peptide prevents degradation by endogenous proteases.
\end{abstract}

